\title{
PHLDA1 expression in ulcerative colitis: A potential role in the management of dysplasia
}

\author{
FUKUICHIRO ORITA $^{1}$, TOSHIAKI ISHIKAWA ${ }^{2}$, MEGUMI ISHIGURO ${ }^{3}$, SATOSHI OKAZAKI $^{2}$, \\ AKIFUMI KIKUCHI $^{1}$, SHINICHI YAMAUCHI ${ }^{1}$, TAKATOSHI MATSUYAMA ${ }^{1}$, \\ MASANORI TOKUNAGA ${ }^{1}$, HIROYUKI UETAKE ${ }^{2}$ and YUSUKE KINUGASA ${ }^{1}$ \\ Departments of ${ }^{1}$ Gastrointestinal Surgery, ${ }^{2}$ Specialized Surgeries and ${ }^{3}$ Translational Oncology, \\ Graduate School of Medical and Dental Sciences, Tokyo Medical and Dental University, Bunkyo-ku, Tokyo 113-8519, Japan
}

Received December 27, 2020; Accepted July 2, 2021

DOI: $10.3892 / \mathrm{mco} .2021 .2354$

\begin{abstract}
Pleckstrin homology-like domain, family A, member 1 (PHLDA1) is a protein involved in cell proliferation, adhesion and migration in colon cancer. In normal large intestinal mucosa, this protein is expressed only in the crypts. By contrast, its expression in adenomas and cancers of the large intestine is spread throughout the glandular ducts, and it has been reported that PHLDA1 may be involved in the process of carcinogenesis. PHLDA1 may also be involved in the pathogenesis of ulcerative colitis (UC). The expression levels of PHLDA1 in tissues from patients with UC were analyzed using immunohistochemistry, and its relationship with the development of UC-associated colorectal cancer (UC-CRC) was examined. Overall, tissue samples from 143 lesions (90 colitis lesions, 39 dysplastic lesions and 14 UC-CRC lesions) were prepared from excised specimens of 49 patients with UC who underwent surgery in Tokyo Medical and Dental University Hospital between January 2004 and December 2017. Subsequently, immunostaining for PHLDA1 was performed. PHLDA1 expression was evaluated in UC-CRC and dysplastic tissues within the entire lesion area on the slide and in colitis over the area of the accompanying duct. The cytoplasmic staining intensity was classified into four levels, and the expression score (0-2 points) was calculated. The median PHLDA1 expression score was 0.295 for colitis, 0.607 for dysplasia and 0.865 for UC-CRC. The dysplasia expression score was significantly higher than the colitis score $(\mathrm{P}<0.001)$, while the UC-CRC expression score was significantly higher than the dysplasia score $(\mathrm{P}=0.003)$. The expression levels of PHLDA1
\end{abstract}

Correspondence to: Dr Toshiaki Ishikawa, Department of Specialized Surgeries, Graduate School of Medical and Dental Sciences, Tokyo Medical and Dental University, 1-5-45 Yushima, Bunkyo-ku, Tokyo 113-8519, Japan

E-mail: ishi.srg2@tmd.ac.jp

Key words: pleckstrin homology-like domain, family A, member 1, ulcerative colitis, dysplasia, ulcerative colitis-associated colorectal cancer in UC cases were higher in colitis, followed by dysplasia and UC-CRC, which suggested that this protein may be involved in the carcinogenesis of UC-CRC. In addition, PHLDA1 immunostaining may help in the diagnosis of dysplasia, which is a type of precancerous lesion.

\section{Introduction}

The worldwide incidence of ulcerative colitis (UC) continues to increase at a significant rate (1). UC is a type of inflammatory bowel disease characterized by periods of inflammatory recurrence and remission events, which are accompanied by cell death and regeneration of the colonic mucosa. It is well known that long-standing UC leads to dysplasia (i.e., precancerous lesions) and colorectal cancer (CRC) and is often a threat to the lives of the patients. The incidence of colorectal dysplasia in patients with UC has been reported to be $1.9 \%$ at 5 years, $5.1 \%$ at 15 years, and $9.2 \%$ at 25 years after the onset of UC (2). The risk of developing UC-associated CRC (UC-CRC) increases $0.5-1 \%$ per year in patients who have had UC for longer than 8-10 years (3). In addition, the prognosis of $\mathrm{CRC}$ is generally poorer in patients with UC than in patients without UC (4). Therefore, surveillance colonoscopy is recommended for the detection of neoplasms in patients with UC. Early detection of UC-CRC is essential for the successful management of long-standing UC (4). However, endoscopic and histologic detection of dysplasia is often difficult due to the presence of inflammatory and subsequent regenerative changes in the colonic mucosa $(3,5)$. For the early detection of dysplasia, it is important to understand the mechanism of CRC development in patients with UC. While chronic inflammation of the colonic mucosa is believed to cause UC-CRC (6), the genetic details of UC-CRC pathogenesis remain unclear (7). In cases of CRC in patients without UC, p53 mutations are generally considered to be involved in the later stages of carcinogenesis (8), while in UC-CRC, p53 mutations have been reported to occur earlier in tumor development $(3,7)$. p53 immunohistochemistry (IHC) is therefore often used for the diagnosis of neoplasms in UC.

In both non-UC-CRC and UC-CRC, the expressions of some genes have different effects on carcinogenesis, but these two diseases also share many common genes (9). 
Previously, our laboratory reported 17 genes associated with distant metastases extracted from microarrays using gene expression data (10), as well as the involvement of the ATF6 in the carcinogenic process of UC (11). ATF6 was rarely expressed in normal mucosa but highly expressed in colon adenomas and CRC. This gene was confirmed to be highly expressed in dysplasia lesions and UC-related cancers. Therefore, we searched for genes that may be involved from the early stages of canceration to the metastatic stage and selected PHLDA1, which encodes for pleckstrin homology-like domain, family A, member 1, which is one of the 17 genes (12). In this study, we focused on the possible involvement of PHLDA 1 in UC carcinogenesis and cancer progression.

\section{Materials and methods}

Identification of PHLDA1. The microarray data used was obtained from a previous study (13). The gene expression data are deposited in the Gene Expression Omnibus (http://www. ncbi.nlm.nih.gov/geo/) under accession number GSE32323.

The gene expression data were analyzed to identify the candidate genes related to distant recurrence. The criteria to select candidate genes were as follows: i) A higher expression level in cancer tissues than in non-cancerous mucosa, and ii) a significantly higher expression level in cancer cells from the recurrence group than in the non-recurrence group. A higher expression was defined as a numerical value 1.5 times greater than those in another group (10). In the microarray analysis, 69 genes were identified that fulfilled the abovementioned criteria. Among 69 genes, 17 genes were found associated with human malignancies. Among the 17 candidate genes, we focused on PHLDA1, which is expressed in adenomas in the early stages of canceration and is also involved in cancer progression.

Patients and samples for the IHC study. A total of 49 consecutive patients with UC who underwent colectomy between January 2004 and December 2017 were included in this study. Overall, 143 lesions were analyzed in the IHC study. The diagnosis was based on surgically resected UC specimens and was made by pathologists who specialize in colon pathology. The pathologic findings of the UC samples were categorized into two groups: The absence of neoplasia group (colitis) and the neoplasia group (dysplasia and UC-CRC).

Samples of non-neoplastic lesions from patients with or without neoplasia were also selected. Samples of neoplasia were selected from each neoplastic lesion present. Neoplastic and non-neoplastic lesions comprised one sample from a single lesion. Table I summarizes the patients' characteristics.

Immunohistochemistry. IHC analysis was performed on 4- $\mu$ m-thick sections cut from formalin-fixed paraffin-embedded tissue blocks obtained from each patient. PHLDA1 and p53-IHC analysis was performed on all samples in this study. All sections were scored independently by two investigators.

IHC for PHLDA1. The streptavidin-biotin method was used for PHLDA1 immunostaining. Xylene is soaked for $10 \mathrm{~min}$ five times. In addition, it is soaked in $100 \%$ ethanol three times for $5 \mathrm{~min}, 95 \%$ ethanol once for $3 \mathrm{~min}, 90 \%$ ethanol once for $3 \mathrm{~min}, 80 \%$ ethanol once for $3 \mathrm{~min}$, and $70 \%$ ethanol once for $3 \mathrm{~min}$. Then it is rehydrated with double-distilled water for 5 min three times.

Antigen retrieval was performed by heating the tissue sections in $\mathrm{pH} 6.0$ citrate buffer in a microwave at $98^{\circ} \mathrm{C}$ for $25 \mathrm{~min}$. Then, sections were then incubated in a solution of $3 \%$ hydrogen peroxide in $100 \%$ methanol for $15 \mathrm{~min}$ at room temperature to quench endogenous peroxidase activity. Next, sections were incubated in a solution of rabbit polyclonal antibody against PHLDA1 (1:250; sc-23866; Santa Cruz Biotechnology) for $15 \mathrm{~min}$ at room temperature and then for a further $16 \mathrm{~h}$ at $4^{\circ} \mathrm{C}$, after which they were labeled with a polymer [Histofine Simple Stain MAXPO (MULTI); Nichirei Bioscience] for $30 \mathrm{~min}$ at room temperature. Staining was visualized after incubation with DAB (0.02\% 3,3'-diaminobenzidine tetrahydrochloride; Nichirei Bioscience) for $10 \mathrm{~min}$ at room temperature. Finally, the slides were counterstained in $1 \%$ Mayer's hematoxylin, after which they were dehydrated in a series of increasing alcohol concentrations, which was followed by xylene immersion, mounting, and coverslipping.

PHLDA1-IHC was evaluated according to a modification of the method previously described by Krajewska et al (14). Positive cytoplasmic staining in colitis, dysplastic, and UC-CRC cells was assessed and scored on the basis of the immunostaining intensity. The cytoplasmic immunostaining intensity of the ductal cells was graded as - (negative), \pm (weak), 1+ (strong), or 2+ (very strong) compared with the stroma cells. The numbers of cells that exhibited each grade of staining intensity were counted independently and totaled. Scores corresponding to the percentage $(\%)$ of cells of each grade of staining intensity were calculated relative to the total number of cells. The score of cytoplasmic staining for PHLDA1 (potentially ranging from 0 to 2) was obtained by summing the product of each percentage score by the corresponding intensity score.

IHC for $p 53$. The streptavidin-biotin method was used for mutated p53 immunostaining. Antigen retrieval was performed by autoclaving the tissues in $\mathrm{pH} 6.0$ citrate buffer at $121^{\circ} \mathrm{C}$ for $15 \mathrm{~min}$.

Endogenous peroxidase activity was quenched using the same method used for PHLDA1-IHC. The sections were sequentially incubated with a polyclonal antihuman p53 antibody (1:200; NCL-L-p53-DO7; Leica Biosystems) for $60 \mathrm{~min}$ at room temperature, MULTI for $30 \mathrm{~min}$ at room temperature, DAB for color development, and 1\% Mayer's hematoxylin for the counterstain. Then, the sections were dehydrated by immersion in a series of alcohol solutions and xylene according to the method used for PHLDA1-IHC. As Shigaki et al (15) reported, the p53 staining pattern in the nucleus was characterized as sporadic, mosaic, nested, and diffuse. Nested or diffuse patterns were considered to represent p53-IHC positivity regardless of the intensity, whereas the sporadic and mosaic patterns were considered to represent p53-IHC negativity.

Statistical analysis. All statistical analyses were performed using EZR (Saitama Medical Center, Jichi Medical University, Saitama, Japan), a graphical user interface for R (The R Foundation for Statistical Computing, Vienna, Austria). More precisely, it is a modified version of $\mathrm{R}$ commander designed to add statistical functions frequently used in biostatistics. For 
Table I. Characteristics of patients with UC.

\begin{tabular}{|c|c|c|c|}
\hline Characteristics & All patients $(n=49)$ & Patients without neoplasia $(n=30)$ & Patients with neoplasia $(n=19)$ \\
\hline Age, years $($ mean \pm SD) & $47.6 \pm 16.9$ & $47.7 \pm 19.5$ & $47.5 \pm 11.2$ \\
\hline \multicolumn{4}{|l|}{ Sex, n (\%) } \\
\hline Male & $30(61.2)$ & $17(56.7)$ & $13(68.4)$ \\
\hline Female & $19(38.8)$ & $13(43.3)$ & $6(31.6)$ \\
\hline \multicolumn{4}{|l|}{ Disease extent, n (\%) } \\
\hline Pancolitis & $49(100.0)$ & $30(61.2)$ & $19(38.8)$ \\
\hline Left-sided colitis & $0(0.0)$ & $0(0.0)$ & $0(0.0)$ \\
\hline Others & $0(0.0)$ & $0(0.0)$ & $0(0.0)$ \\
\hline \multicolumn{4}{|c|}{ Past history of 5-ASA use, n (\%) } \\
\hline Yes & $23(46.9)$ & $11(47.8)$ & $12(52.2)$ \\
\hline No & $26(53.1)$ & $19(73.1)$ & $7(26.9)$ \\
\hline \multicolumn{4}{|c|}{ Past history of steroid use, n (\%) } \\
\hline Yes & $32(65.3)$ & $24(75.0)$ & $8(25.0)$ \\
\hline No & $17(34.7)$ & $6(35.3)$ & $11(64.7)$ \\
\hline \multicolumn{4}{|l|}{ Number of samples, n (\%) } \\
\hline Colitis & $90(62.9)$ & $64(71.1)$ & $26(28.9)$ \\
\hline Dysplasia & $39(27.3)$ & $0(0.0)$ & $39(100.0)$ \\
\hline UC-CRC & $14(9.8)$ & $0(0.0)$ & $14(100.0)$ \\
\hline
\end{tabular}

UC, ulcerative colitis; 5-ASA, 5-aminosalicylate; UC-CRC, UC-associated colorectal cancer.

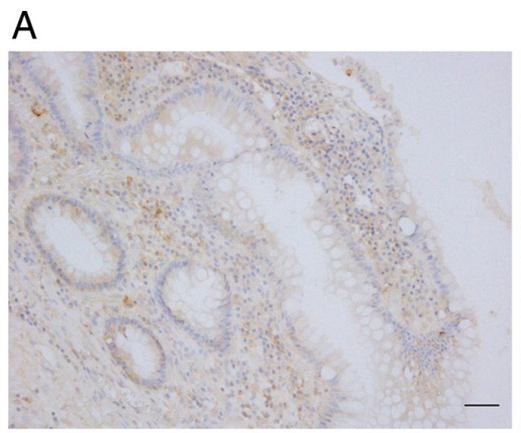

B

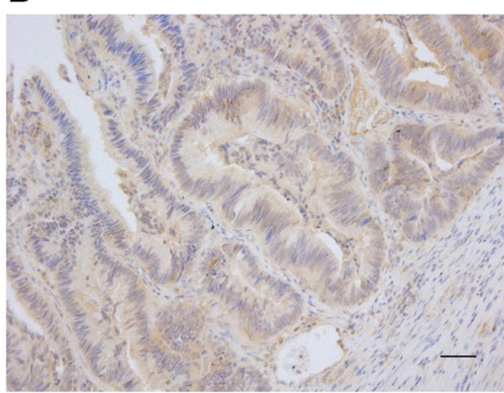

C

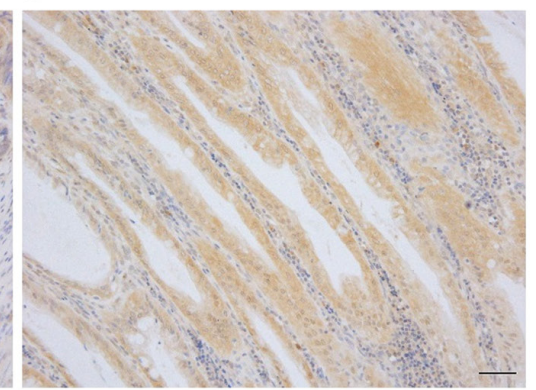

Figure 1. Pleckstrin homology-like domain, family A, member 1 expression in UC samples. (A) In colitis, the cytoplasmic staining of ductal cells was poor or equivalent to the staining of stromal cells. The intensities of these cells were evaluated as - or \pm , respectively. (B) In dysplasia, the cytoplasmic staining of ductal cells was stronger than the stromal cell staining. The intensities of these cells were evaluated as + . (C) In UC-associated colorectal cancer, the cytoplasmic staining of ductal cells was strongly or markedly greater than that of stromal cells. The intensities of these cells were evaluated as + or $2+$, respectively. Scale bar, $50 \mu \mathrm{m}$. UC, ulcerative colitis.

categorical data, the significance of between-group differences was estimated using the Fisher's exact test and $\chi^{2}$ test, as appropriate. For continuous variables, descriptive statistics of the mean, median, and range were calculated, and the significance of between-group differences was estimated using the Kruskal-Wallis test as appropriate. The Holm method was used to correct for significant differences during multiple comparisons. A P value $<0.05$ was considered statistically significant.

\section{Results}

PHLDA1 expression in UC samples. Of the 49 subjects, 30 exhibited no neoplastic lesions and 19 had neoplasia. The pathologies of the 143 lesions were as follows: 90 colitis,
39 dysplasia, and 14 UC-CRC. The representative results of the PHLDA1-IHC staining in UC samples are shown in Fig. 1. The median PHLDA1-IHC score of the 143 UC samples was 0.40 (range, 0.00-1.43). The median PHLDA1-IHC score in the 39 dysplasia samples was 0.607 (range, 0.03-1.32), which was significantly higher $(\mathrm{P}<0.001)$ than that in the 90 colitis samples (median, 0.29, range, 0-1.29). The median PHLDA1-IHC score in the 14 UC-CRC samples was 0.865 (range, 0.45-1.42), which was significantly higher $(\mathrm{P}=0.003)$ than that in the dysplasia samples. The PHLDA1-IHC score tended to increase as the UC cell variant progressed (Fig. 2).

PHLDA1-IHC and p53-IHC positivity rates in UC samples. The PHLDA1-IHC scores were divided into two groups 


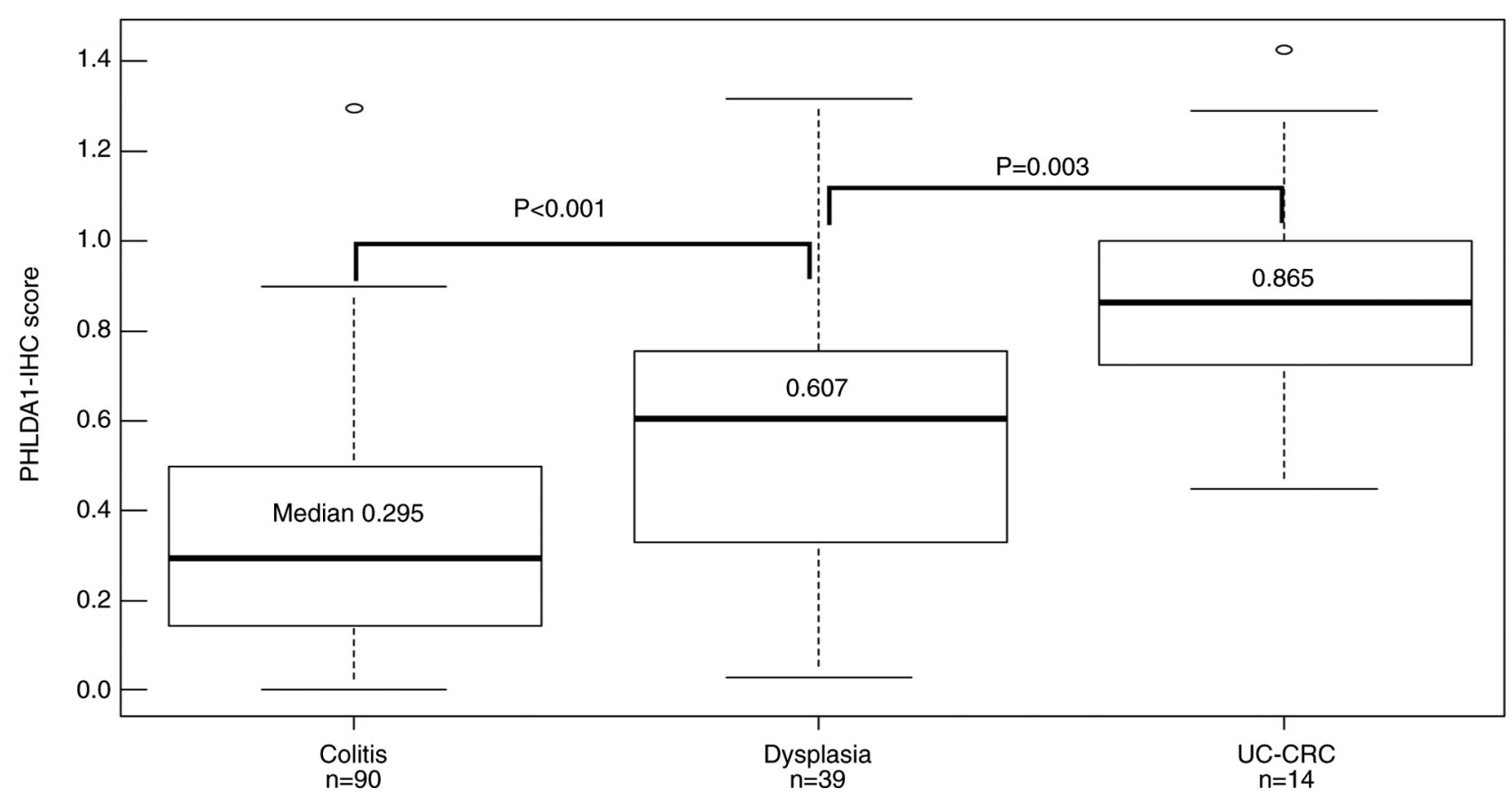

Figure 2. PHLDA1-IHC scores of the UC samples according to histological category. The median PHLDA1-IHC score of the 39 dysplasia samples was 0.607 (range, 0.03-1.32), which was significantly higher $(\mathrm{P}<0.001)$ than that of the 90 colitis samples (median, 0.295; range, 0-1.29). The median PHLDA1-IHC score of the $14 \mathrm{UC}-\mathrm{CRC}$ samples was 0.865 (range, $0.45-1.42$ ), which was significantly higher $(\mathrm{P}=0.003)$ than that of the dysplasia samples. The Kruskal-Wallis test was performed to compare these three groups. The Holm method was used to correct for significant differences during multiple comparisons. PHLDA1, pleckstrin homology-like domain, family A, member 1; IHC, immunohistochemistry; UC, ulcerative colitis; UC-CRC, UC-associated colorectal cancer.

as follows: the PHLDA1-positive (PHLDA1-IHC score $\geq 0.603 \mathrm{n}=47$ ) and PHLDA1-negative groups (PHLDA1-IHC score $\leq 0.603, \mathrm{n}=96$ ). The cutoff value using the receiver operating characteristic curve calculated from colitis and dysplasia was 0.603 , which is the threshold at which the sum of sensitivity and specificity is maximized (Fig. 3). The status of p53-IHC was classified as either p53-positive $(n=25)$ or p53-negative $(n=118)$ according to the method reported by Shigaki et al (15). The PHLDA1 and $\mathrm{p} 53$ positivity rates in the UC samples are shown in Fig. 4. The PHLDA1 positivity rates in neoplastic tissues were higher than that in non-neoplastic tissues. Notably, an obvious difference was observed in the PHLDA1-IHC positivity rates between UC-CRC (78.6\%) and dysplasia samples $(53.8 \%, \mathrm{P}<0.001)$ and between UC-CRC and colitis samples $(15.6 \%, \mathrm{P}<0.001)$. On the contrary, the positivity of PHLDA1 was not statistically significant $(\mathrm{P}=0.067)$ than that of $\mathrm{p} 53$ in dysplasia samples.

Accuracy of PHLDA1 and p53-IHC for a diagnosis of dysplasia. In 129 samples including dysplasia $(\mathrm{n}=39)$ and colitis samples $(n=90)$, we evaluated the accuracy of PHLDA1and p53-IHC for the discrimination of dysplasia from the inflammatory mucosa of UC. The positive predictive value (PPV) of PHLDA1-IHC was $60.0 \%$, which was clearly lower than that of p53 (70.6\%). The negative predictive value (NPV) of PHLDA1-IHC (80.9\%) was relatively higher than that of p53 (75.9\%) (Table II). The sensitivity of PHLDA1-IHC was $53.8 \%$, which was clearly higher than that of p53 (30.8\%). The specificity of PHLDA1-IHC (84.4\%) was relatively lower than that of p53 (94.4\%). Twenty-five (17.5\%) lesions were PHLDA1 positive and p53 negative, of which 14 were dysplasia (56.0\%). Seven (4.9\%) lesions were PHLDA1 nega-

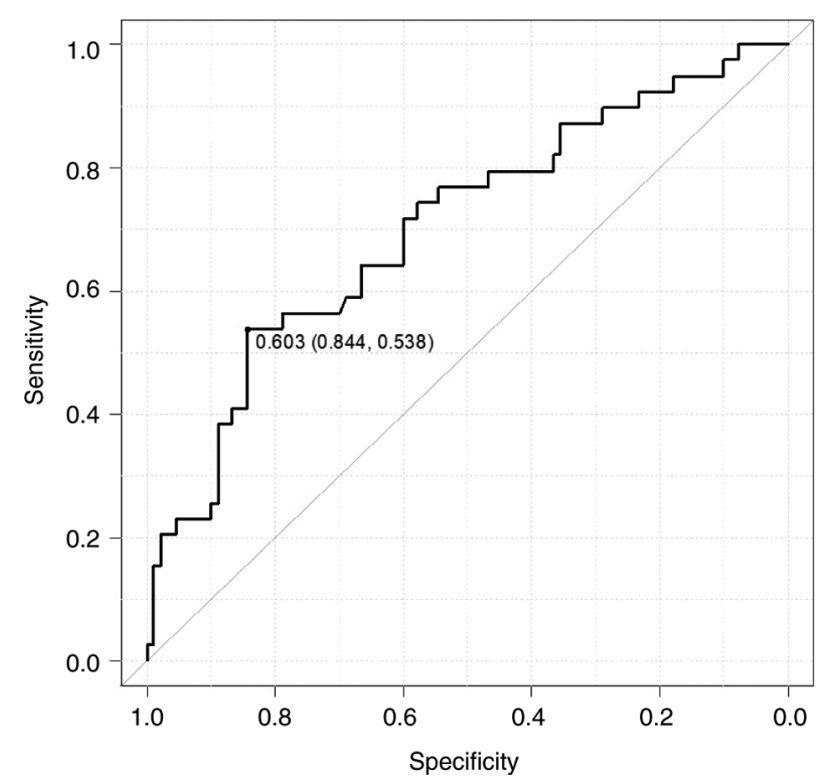

Figure 3. ROC curve. The cutoff value for colitis and dysplasia calculated using the ROC curve was 0.603 (specificity, 0.844; sensitivity, 0.538). ROC, receiver operating characteristic.

tive and p53 positive, of which five were dysplasia (71.4\%) (Table III). No significant differences were observed between the PHLDA1 positive and p53 negative group and PHLDA1 negative and $\mathrm{p} 53$ positive group in age $(\mathrm{P}=0.65)$, gender $(\mathrm{P}=0.64)$, location (proximal/distal; $\mathrm{P}=1.00)$, and reason for surgery $(\mathrm{P}=1.00)$. The $\mathrm{UC}$ lesions were divided into the proximal (cecum, ascending colon and transverse colon) and distal parts (descending colon, sigmoid colon and rectum). The 


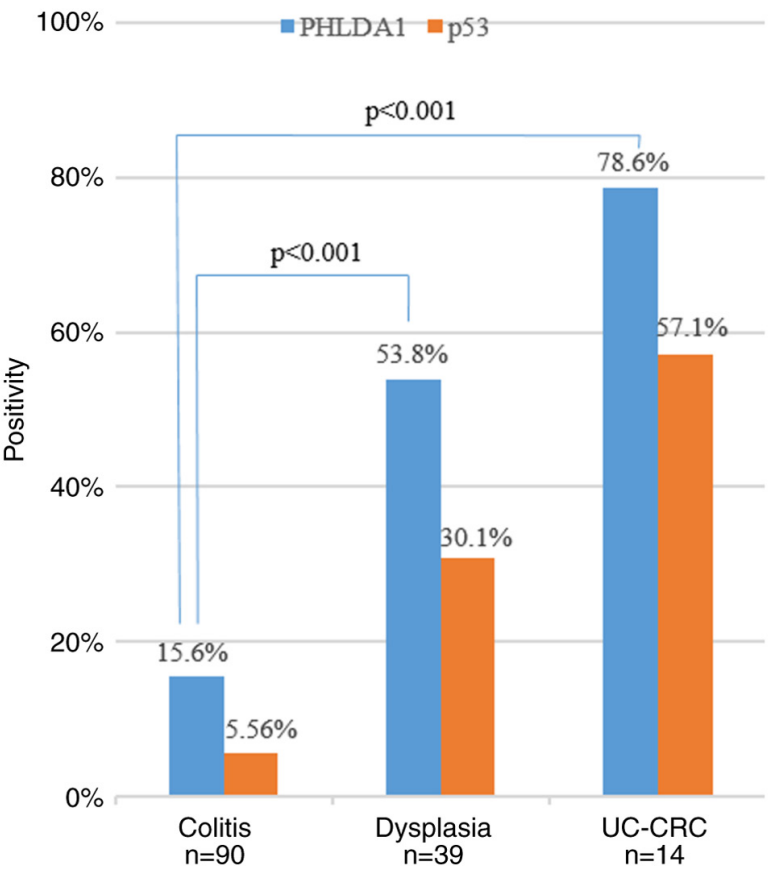

Figure 4. PHLDA1-IHC and p53-IHC positivity rates according to the histological status. The PHLDA1 positivity rate in the neoplastic tissues was higher than that in the non-neoplastic tissues. Notably, an obvious difference was observed in the PHLDA1-IHC positivity rate between the UC-CRC (78.6\%) and dysplasia samples $(53.8 \%$; $\mathrm{P}<0.001)$, and between the UC-CRC and colitis samples $(15.6 \% ; \mathrm{P}<0.001)$. Fisher's test was used to compare the ratio differences between the two groups. PHLDA1, pleckstrin homology-like domain, family A, member 1; IHC, immunohistochemistry; UC-CRC, ulcerative colitis-associated colorectal cancer.

proximal parts included 53 lesions, of which 44 were colitis, and 9 were dysplasia. Ninety lesions were found in the distant parts, of which 46 were colitis, 30 were dysplasia, and 14 were UC-CRC. PHLDA1 and p53 had no significant differences in positivity rate in dysplasia in the proximal and distal regions (Table SI). On the other hand, in colitis, PHLDA1 showed a significant difference in positivity rate (Table SII). In the sites in the proximal region, the PPV of PHLDA1-IHC was 77.8\%, which was clearly higher than that of p53 (66.6\%). The NPV of PHLDA1-IHC (95.5\%) was relatively higher than that of p53 (86.0\%) (Table SIII). The sensitivity of PHLDA1-IHC was $77.8 \%$, which was clearly higher than that of p53 (22.2\%). The specificity of PHLDA1-IHC $(95.5 \%)$ was lower than that of p53 (97.7\%). In the sites in the distal region, the PPV of PHLDA1-IHC was $53.8 \%$, which was clearly lower than that of p53 (71.4\%). The NPV of PHLDA1-IHC (68.0\%) was higher than that of p53 (67.7\%) (Table SIV). The sensitivity of PHLDA1-IHC was $46.7 \%$, which was clearly higher than that of p53 (33.3\%). The specificity of PHLDA1-IHC (73.9\%) was relatively lower than that of p53 $(91.3 \%)$. We found no significant differences in the positivity rates of PHLDA1 and p53 between the colitis part of the patients with UC with dysplasia and/or UC-CRC $(\mathrm{P}=0.24)$ and those without dysplasia and UC-CRC $(\mathrm{P}=0.18)$ (Table SV).

\section{Discussion}

In this study, we demonstrated that PHLDA1 is highly expressed in dysplastic and UC-CRC lesions.
Table II. Accuracy of PHLDA1-IHC and p53-IHC for diagnosis of dysplasia.

\begin{tabular}{lcccc}
\hline Outcome & Dysplasia, $n$ & Colitis, $n$ & PPV, \% & NPV, \% \\
\hline PHLDA1 & & & & \\
Positive & 21 & 14 & 60.0 & 80.9 \\
Negative & 18 & 76 & & \\
p53 & & & & \\
Positive & 12 & 5 & 70.6 & 75.9 \\
Negative & 27 & 85 & & \\
\hline
\end{tabular}

PHLDA1, pleckstrin homology-like domain, family A, member 1; IHC, immunohistochemistry; PPV, positive predictive value; NPV, negative predictive value.

Table III. Dysplasia rate and combination of PHLDA1-IHC and p53-IHC status.

\begin{tabular}{lll}
\hline Outcome & p53 positive & p53 negative \\
\hline PHLDA1 positive & $70.0 \%(7 / 10)$ & $56.0 \%(14 / 25)$ \\
PHLDA1 negative & $71.4 \%(5 / 7)$ & $14.9 \%(13 / 87)$ \\
\hline
\end{tabular}

PHLDA1, pleckstrin homology-like domain, family A, member 1; IHC, immunohistochemistry.

PHLDA1 was first identified as a potential transcription factor that is required for Fas expression and activation-induced apoptosis in mouse T cell hybridomas (1). The PHLDAl is located on 12q21.2 and contains PHL domains; these domains interact with membrane components, which elicit a variety of cellular responses, through which it participates in cell signaling transduction, vesicular trafficking, and cytoskeletal rearrangement $(16,17)$. PHLDA1 is predominantly expressed in the cytoplasm (18-21), and its expression is induced by different stimuli, such as estrogens (22), growth factors (23), differentiation factors (24), and endoplasmic reticulum stress-inducing agents (25). PHLDA1 is also associated with various biological processes, such as cell apoptosis, cell proliferation, and differentiation.

In terms of the relationship between PHLDA1 and cancer, it has been reported that an osteosarcoma cell line with a high metastatic potential had increased PHLDA1 expression (26). It has also been found that PHLDA1 has an apoptosis-suppressing effect in oral cancer $(27,28)$. In addition, the relationship between breast cancer and PHLDA1 has been well studied, and E2 and TNF- $\alpha$ are considered promoters of PHLDA1. However, PHLDA1 in breast cancer is a tumor suppressor gene (22).

As for the relationship between PHLDAl and the colonic mucosa, $P H L D A 1$ is only expressed in undifferentiated basal cells in the crypts. It is also highly expressed in colorectal adenoma and carcinoma cells, and high expression of PHLDA1 has been shown to increase migration ability, anchorageindependent growth, and cell-matrix adhesion ability (12).

In this study, we showed that the PHLDA1-IHC score is significantly elevated as the UC cell variant progresses. Since 
no studies have been retrieved showing that the canceration of UC involves the proliferation of undifferentiated basal cells, PHLDAl was presumed to also be involved in the canceration of UC. However, this study could not clarify whether this gene was an initiating factor or a promoting factor in the canceration of UC. Furthermore, the presence of p53 gene mutation suggests the presence of dysplasia, a precancerous lesion.

p53-IHC is widely used as an auxiliary diagnostic method, but the correct diagnosis rate of dysplasia is low, and the sensitivity is believed to be $11-40 \%$ (29), which was similar to the findings in this study. The diagnosis of high p53 protein expression by IHC does not always correspond to the p53 gene mutation. In other words, p53 IHC alone may overlook dysplasia. The detection of promising biomarkers for the diagnosis of dysplasia could impact the clinical management of patients with $\mathrm{UC}$, who are at a higher risk for cancer development (3). This is because if high-grade dysplasia develops, those patients will be treated by total colectomy, which may significantly reduce the patient's quality of life. Since p53 IHC has a high PPV (70.6\%), it is probable that dysplasia can be diagnosed in positive cases. Moreover, since the specificity of p53 IHC is as high as $94.4 \%$, it can be reasonably used as an auxiliary diagnosis. However, due to its low sensitivity (30.8\%), many cases of dysplasia were present among those who were diagnosed as negative. Since PHLDA1-IHC is more sensitive than p53-IHC, 56\% of dysplasia cases that were diagnosed as negative by p53-IHC were positive (Table III). Since PHLDA1-IHC is more sensitive than p53-IHC, it is more useful as a screening diagnosis to ensure diagnostic accuracy, and when combined with the highly sensitive PHLDA1, it may be a useful auxiliary diagnostic marker. Therefore, it may be possible to include, by PHLDA1-IHC, those cases that have been determined to be negative by p53-IHC and that are difficult to determine pathologically. In addition, PHLDA1 had a high PPV, which suggests that it may be more effective, especially for dysplasia that occurred in the proximal colon (Table SIII).

However, one limitation of this study includes its small sample size. This is because we collected multiple samples (pathological sections) from a single patient. However, as far as possible, we collected different and individual tissues from that patient, including UC-CRC, dysplasia, inflammatory, and other tissues. Moreover, the histological type was as different as possible in the tissues collected from that patient, e.g., UC-CRC tissue and cells. We collected and examined dysplasia from the section that appeared to be strongly atypical as well as from the section where cell atypia was likely to be weak, the section with strong inflammation, and the section with weak inflammation. Furthermore, this study was only verified by immunostaining since no blood and stool samples were collected, which could otherwise further verify PHLDA1 expression. A future prospective study that includes a larger number of endoscopic biopsy samples along with long-term surveillance with endoscopy is needed.

In conclusion, PHLDAl was believed to be involved in UC carcinogenesis, and PHLDA1-IHC has been suggested to contribute to the diagnosis of dysplasia when used in combination with p53-IHC.

\section{Acknowledgements}

The authors would like to thank Mrs. Yoko Takagi and Mrs. Junko Inoue (Department of Specialized Surgeries,
Graduate School of Medical and Dental Sciences, Tokyo Medical and Dental University, Bunkyo-ku, Tokyo 113-8519, Japan) for their excellent technical assistance.

\section{Funding}

No funding was received.

\section{Availability of data and materials}

The datasets used and/or analyzed during the current study are available from the corresponding author on reasonable request.

\section{Authors' contributions}

FO was responsible for the study conception and design, the data collection and analysis, performing the experiments, writing the manuscript and the statistical analysis. TI, MI and SO contributed to the study conception and design, data analysis, and data interpretation, and provided technical and material support. SY, AK, TM, MT, HU and YK contributed to the study conception, data interpretation, and critically revised the manuscript. FO and TI confirmed the authenticity of all the raw data. All authors read and approved the final manuscript.

\section{Ethics approval and consent to participate}

The present study was conducted in accordance with the Declaration of Helsinki and its later amendments or comparable ethical standards. The study protocol was approved by the Institutional Review Board of Tokyo Medical and Dental University (approval. no. D2000-831; date, January 1, 2000 to June 30, 2025; Tokyo, Japan), and written informed consent was obtained from all the patients before enrollment.

\section{Patient consent for publication}

Not applicable.

\section{Competing interests}

The authors declare that they have no competing interests.

\section{References}

1. Molodecky NA, Soon IS, Rabi DM, Ghali WA, Ferris M, Chernoff G, Benchimol EI, Panaccione R, Ghosh S, Barkema HW and Kaplan GG: Increasing incidence and prevalence of the inflammatory bowel diseases with time, based on systematic review. Gastroenterol 142: 46-54, 2012.

2. Jess T, LoftusEV Jr, Velayos FS, Harmsen WS, Zinsmeister AR, Smyrk TC, Tremaine WJ, Melton LJ 3rd, Munkholm P and Sandborn WJ: Incidence and prognosis of colorectal dysplasia in inflammatory bowel disease: A population-based study from olmsted county, Minnesota. Inflamm Bowel Dis 12: 669-676, 2006.

3. Chen R, Lai LA, Brentnall TA and Pan S: Biomarker for colitis-associated colorectal cancer. World J Gastroenterol 22: 7882-7891, 2016.

4. Watanabe T, Konishi T, Kishimoto J, Kotake K, Muto T, Sugihara K; Japanese Society for Cancer of the Colon and Rectum: Ulcerative colitis-associated colorectal cancer shows a poorer survival than sporadic cancer: A nationwide Japanese study. Inflamm Bowel Dis 17: 802-808, 2011.

5. DeRoche TC, Xiao SY and Liu X: Histological evaluation in ulcerative colitis. Gastroenterol Rep (Oxf) 2: 178-192, 2014. 
6. Okamoto R and Watanabe M: Role of epithelial cells in the pathogenesis and treatment of inflammatory bowel disease. J Gastroenterol 51: 11-21, 2016.

7. Triantafillidis JK, Nasioulas G and Kosmidis PA: Colorectal cancer and inflammatory bowel disease: Epidemiology, risk factors, mechanisms of development and prevention strategies. Anticancer Res 29: 2727-2737, 2009.

8. Vogelstein B, Fearson ER, Hamilton SR, Kern SE, Preisinger AC Leppert M, Nakamura Y, White R, Smits AM, and Bos JL: Genetic alterations during colorectal-tumor development. N Engl J Med 319: 525-532, 1988.

9. Ullmam TA and Itzkowitz SH: Intestinal inflammation and cancer. Gastroenterology 140: 1807-1816, 2011.

10. Takahashi H, Ishikawa T, Ishiguro M, Okazaki S, Mogushi K, Kobayashi H, Iida S, Mizushima H, Tanaka H, Uetake H and Sugihara K: Prognostic significance of Traf2- and Nck- interacting kinase (TNIK) in colorectal cancer. BMC Cancer 15: 794, 2015.

11. Hanaoka M, Ishikawa T, Ishiguro M, Tokura M, Yamauchi S Kikuchi A, Uetake H, Yasuno M and Kawano T: Expression of ATF6 as a marker of pre-cancerous atypical change in ulcerative colitis-associated colorectal cancer. A potential role in the management of dysplasia. J Gastroenterol 53: 631-641, 2018.

12. Sakthianandeswaren A, Christie M, D'Andreti C, Tsui C, Jorissen RN, Li S, Fleming NI, Gibbs P, Lipton L, Malaterre $\mathrm{J}$, et al: PHLDA1 expression marks the putative epithelial stem cells and contributes to intestinal tumorigenesis. Cancer Res 71: 3709-3719, 2011.

13. Khamas A, Ishikawa T, Shimokawa K, Mogushi K, Iida S, Ishiguro M, Mizushima H, Tanaka H, Uetake H and Sugihara K: Screening for epigenetically masked genes in colorectal cancer using 5-Aza-2'-deoxycytidine, microarray and gene expression profile. Cancer Genomics Proteomics 9: 67-75, 2012.

14. Krajewska M, Krajewski S, Epstein JI, Shabaik A, Sauvageot J, Song K, Kitada S and Reed JC: Immunohistochemical analysis of bcl-2, bax, bcl-X, and mcl-1 expression in prostate cancers. Am J Pathol 148: 1567-1576, 1996.

15. Shigaki K, Mitomi H, Fujimori T, Ichikawa K, Tomita S, Imura J, Fujii S, Itabashi M, Kameoka S, Sahara R and Takenoshita S: Immunohistochemical analysis of chromogranin A and p53 expressions in ulcerative colitis-associated neoplasia: Neuroendocrine differentiation as an early event in the colitis-neoplasia sequence. Hum Pathol 44: 2393-2399, 2013.

16. Lemmon MA and Ferguson KM: Signal-dependent membrane targeting by pleckstrin homology $(\mathrm{PH})$ domains. Biochem J 15 : $1-18,2000$.

17. Scheffzek K and Welti S: Pleckstrin homology (PH) like domains - versatile modules in protein-protein interaction platforms. FEBS Lett 586: 2662-2673, 2012.

18. Hinz T, Flindt S, Marx A, Janssen O and Kabelitz D: Inhibition of protein synthesis by the $\mathrm{T}$ cell receptor-inducible human TDAG51 gene product. Cell Signal 13: 345-352, 2001.
19. Neef R, Kuske MA, Pröls E and Johnson JP: Identification of the human PHLDA1/TDAG51 gene: Down-regulation in metastatic melanoma contributes to apoptosis resistance and growth deregulation. Cancer Res 62: 5920-5929, 2002.

20. Nagai MA, Fregnani JH, Netto MM, Brentani MM and Soares FA: Down-regulation of PHLDA1 gene expression is associated with breast cancer progression. Breast Cancer Res Treat 106: 49-56, 2007.

21. Zhao P, Lu Y and Liu L: Correlation of decreased expression of PHLDA1 protein with malignant phenotype of gastric adenocarcinoma. Int J Clin Exp Pathol 8: 5230-5235, 2015.

22. Marchiori AC, Casolari DA and Nagai MA: Transcriptional up-regulation of PHLDA1 by 17beta-estradiol in MCF-7 breast cancer cells. Braz J Med Biol Res 41: 579-582, 2008.

23. Toyoshima Y, Karas M, Yakar S, Dupont J, Helman L and LeRoith D: TDAG51 mediates the effects of insulin-like growth factor I (IGF-I) on cell survival. J Biol Chem 279: 25898-25904, 2004.

24. Hossain GS, van Thienen JV, Werstuck GH, Zhou J, Sood SK, Dickhout JG, de Koning AB, Tang D, Wu D, Falk E, et al: TDAG51 is induced by homocysteine, promotes detachment-mediated programmed cell death, and contributes to the cevelopment of atherosclerosis in hyperhomocysteinemia. J Biol Chem 278: 30317-3027, 2003.

25. Gomes I, Xiong W, Miki T and Rosner MR: A proline- and glutamine-rich protein promotes apoptosis in neuronal cells. J Neurochem 73: 612-622, 1999.

26. Ren L, Mendoza A, Zhu J, Briggs JW, Halsey C, Hong ES, Burkett SS, Morrow J, Lizardo MM, Osborne T, et al: Characterization of the metastatic phenotype of a panel of established osteosarcoma cells. Oncotarget 6: 29469-29481, 2015.

27. Murata T, Sato T, Kamoda T, Moriyama H, Kumazawa Y and Hanada N: Differential susceptibility to hydrogen sulfide-induced apoptosis between PHLDA1-overexpressing oral cancer cell lines and oral keratinocytes: Role of PHLDA1 as an apoptosis suppressor. Exp Cell Res 320: 247-257, 2014.

28. Coutinho-Camillo CM, Lourenço SV, Nonogaki S, Vartanian JG, Nagai MA, Kowalski LP and Soares FA: Expression of PAR-4 and PHLDA1 is prognostic for overall and disease-free survival in oral squamous cell carcinomas. Virchows Arch 463: 31-39, 2013.

29. Taylor HW, Boyle M, Smith SC, Bustin S and Williams NS: Expression of p53 in colorectal cancer and dysplasia complicating ulcerative colitis. Br J Surg 80: 442-444, 1993. 